\title{
Os Efeitos da Idade na Seleção de Carga Perceptual
}

\author{
Vanessa Coelho de Sousa ${ }^{1}$ \\ Joaquim Carlos Rossini \\ Universidade Federal de Uberlândia
}

\begin{abstract}
RESUMO - Algumas pesquisas sugerem a existência de momentos distintos de maturação do sistema atentivo. O presente estudo investigou os padrões de seleção da informação visual através de uma tarefa de carga perceptual executada por três grupos etários: crianças, adultos e idosos. De maneira geral os resultados obtidos indicaram uma diminuição da eficiência no processo de seleção da informação em condições de baixa carga perceptual na população de idosos e uma diminuição da eficiência da seleção em condições de alta e baixa carga perceptual na população composta por crianças. Estes resultados sugerem a existência de padrões específicos na seleção da informação visual em função da idade e da carga perceptual a ser processada pelo sistema visual.
\end{abstract}

Palavras-Chaves: atenção visual; carga perceptual; diferenças etárias.

\section{Effects of Aging on the Selection of Perceptual Load}

\begin{abstract}
Some studies suggest the existence of distinct maturation moments of the attentional system. This study investigated the selection patterns of visual information through a perceptual load task performed by three age groups: children, adults and elderly people. In general, the obtained results indicate a decrease of the efficiency in the process of information selection under low perceptual load in the group of elderly people, and a decrease of the selection efficiency under conditions of high and low perceptual load in the group composed by children. The results suggest the existence of specific patterns in the selection of visual information in relation to age and the perceptual load to be processed by the visual system.
\end{abstract}

Keywords: visual attention; perceptual load; age differences.

Os processos atentivos de seleção e análise da informação exercem um papel crucial na interação do individuo com o ambiente circundante, pois é com esses processos que buscamos os estímulos relevantes para o nosso dia a dia e descartamos aqueles irrelevantes. Existem algumas controvérsias entre as teorias que tentam explicar como se dá essa seleção e o processamento mental dos estímulos ambientais, principalmente a respeito do momento do processamento no qual esta seleção ocorre. Alguns autores sugerem que essa seleção se dá de forma precoce (Broadbent, 1958; Treisman, 1964), ou seja, logo após a ocorrência do registro dos estímulos no nível sensorial, enquanto que outros autores propõem uma seleção mais tardia da informação (Deutsch \& Deutsch, 1963), no qual a informação seria processada ou descartada após um processamento cognitivo sucessivo .

Recentemente, algumas pesquisas sugerem que o mecanismo de seleção a ser utilizado pelo sistema cognitivo (precoce ou tardio) pode ser definido pela carga perceptual de uma cena visual. Este mecanismo de seleção ficou conhecido como Modelo da Carga Perceptual (Lavie, 1995; Lavie \& Cox, 1997; Lavie \& Tsal, 1994) e considera, em linhas gerais, que a carga perceptual é determinante para o tipo de seleção dos estímulos contidos em um campo visual (seleção precoce ou tardia). Este modelo sugere que o processo perceptual é limitado, e seleciona de forma eficiente os estímulos relevantes dos irrelevantes até o limite da sua

1 Endereço para correspondência: Universidade Federal de Uberlândia, Instituto de Psicologia, Campus Umuarama. Av. Pará, 1720 - Bloco 2C 29. Uberlândia, MG. CEP: 38405-320. Fone: 3218-2701. E-mail: vanessacoelho@mestrado.ufu.br capacidade. Assim, quando a carga perceptual do ambiente é menor que a capacidade disponível do sistema atentivo, este processa os estímulos selecionando-os de forma semântica, em um estágio tardio de processamento de informação. Porém, quando a capacidade do sistema atentivo é excedida, os elementos relevantes passam a ser selecionados com base nas suas características físicas mais proeminentes, em um processo precoce de descarte da informação irrelevante.

Lavie (1995) investigou esta hipótese manipulando diferentes cargas perceptuais e supôs que quanto maior a carga perceptual menor seria a interferência de estímulos flanqueadores (flankers) apresentados fora da área relevante para a tarefa dos participantes, seus resultados confirmaram esta hipótese. Houve uma redução do efeito das letras flanqueadoras com o aumento da carga perceptual, o que sugere dois aspectos da seleção. $\mathrm{O}$ primeiro aspecto diz respeito à mobilização dos recursos atentivos. Em situações de alta carga perceptual, os recursos seriam mobilizados e utilizados no processamento da informação relevante, restando poucos recursos para o processamento da informação irrelevante (estímulos flanqueadores). O segundo aspecto diz respeito propriamente ao mecanismo de seleção da informação, que em condições de alta carga perceptual executa o processo de seleção baseado nas características físicas dos estímulos, realizando assim uma seleção precoce da informação. Por outro lado, em condições de baixa carga perceptual os recursos seriam mobilizados e utilizados no processamento de toda a informação apresentada na cena visual, uma vez que há recursos atentivos suficientes para isto. Nesta situação, o mecanismo de seleção da informação estaria baseado nas 
características semânticas dos estímulos em um processo tardio de seleção. Desta forma, o modelo da Carga Perceptual (precoce e tardio) alia o mecanismo de seleção à quantidade de informação presente no ambiente, aumentado de certa forma a flexibilidade do processo de seleção da informação.

Uma questão ainda pouco investigada diz respeito às características deste mecanismo de seleção ao longo das fases do desenvolvimento (crianças, adultos e idosos). Alguns autores (Maylor \& Lavie, 1998; Huang-Pollock, Carr, \& Nigg, 2002; Pesce, Guidetti, Baldari, Tessitore \& Capranica, 2005) sugerem a existência de diferenças nos desempenhos de crianças, adultos jovens e idosos em tarefas atentivas. Algumas teorias que tentam explicar essas diferenças postulam acerca de algumas possibilidades, tais como: 1) uma possível redução, com a idade, da energia psíquica disponível para os processos cognitivos; 2) uma possível redução significativa na capacidade inibitória, o que acarretaria déficits atentivos decorrentes de uma diminuição da capacidade de filtragem da informação e conseqüentemente um aumento de intrusões e distrações ocasionadas por estímulos irrelevantes (Hasher \& Zacks, 1988); 3) ou ainda déficits do sistema atentivo de idosos associados à redução do tamanho do campo atentivo (Cerella, 1985).

No que se refere à primeira fase do desenvolvimento do sistema atentivo, Huang-Pollock, Carr e Nigg (2002) sugeriram que há uma consolidação relativamente precoce dos processos automáticos de seleção centrados nas características do objeto, em comparação aos processos semânticos de seleção centrados no significado do objeto, ou seja, que os mecanismos de seleção baseados em processos cognitivos superiores (seleção tardia) e os processos de seleção baseados em características centradas no objeto, como tamanho, movimento e cor (seleção precoce) apresentam tempos distintos de maturação e, por conseguinte, estariam mais disponíveis em certos momentos do desenvolvimento do sujeito que em outros, o que influenciaria significativamente a maneira com a qual os indivíduos processam e selecionam as informações relevantes contidas no ambiente (Huang-Pollock, Carr, \& Nigg, 2002).

Para analisar estes mecanismos de seleção em diferentes grupos etários Maylor e Lavie (1998) realizaram dois experimentos que investigaram o desempenho de adultos jovens e idosos em uma tarefa de seleção atentiva e carga perceptual. De modo geral, os resultados desses autores indicaram tanto uma diminuição na acurácia quanto um aumento no tempo de reação no grupo composto por idosos em comparação ao grupo de adultos jovens. Mais especificamente, os resultados do primeiro experimento deste trabalho corroboraram o modelo proposto por Lavie (1995) e sugerem que a capacidade de processamento diminui com o aumento da idade. Estes resultados mostraram um efeito significativamente maior dos estímulos flanqueadores para os participantes idosos em comparação ao grupo de participantes jovens. Os resultados também mostraram que o efeito negativo dos estímulos flanqueadores sofre um declínio mais acentuado no grupo de idosos quando comparado a grupo de jovens.

Um aspecto importante apontado por alguns autores (Plude, Enns, \& Brodeur, 1994; Huang-Pollock, Carr, \& Nigg, 2002) é que a capacidade de seleção precoce da informação, centrada nas características físicas do objeto, seria adquirida rapidamente durante a infância e apresentaria uma probabilidade menor de regredir em uma idade avançada. Por outro lado, a capacidade de seleção tardia centrada nas representações semânticas dos objetos, seria adquirida em fases mais tardias do desenvolvimento cognitivo, ficando mais suscetível à regressão durante a velhice.

Com isso, a presente pesquisa teve como objetivo avaliar possíveis diferenças nos processos atentivos em três fases da vida: crianças, adultos jovens e idosos. Para tanto, foi delineado um experimento similar ao realizado por Huang-Pollock e cols. (2002) no qual os grupos investigados foram submetidos a uma tarefa de seleção atentiva e carga perceptual. A análise em conjunto dos três grupos etários permitiu-nos uma discussão acerca de possíveis diferenças etárias na seleção e processamento da informação visual.

\section{Método}

\section{Participantes}

Este experimento contou com a participação voluntária de 30 sujeitos divididos em três grupos etários: crianças, adultos jovens e idosos. Dez crianças de 9 a 13 anos $(\mathrm{M}=11$; $\mathrm{DP}=$ $1,4)$ com tempo médio de estudo de 6 anos; 10 adultos jovens de 21 a 29 anos $(M=23,6 ; \mathrm{DP}=2,5)$ e média de estudo de 14,8 anos; e 10 idosos de 62 a 78 anos $(\mathrm{M}=66, \mathrm{DP}=5)$ e média de tempo de estudo igual a 10 anos. Todos sem queixa clínica, com visão normal ou corrigida. O grupo de crianças era composto por estudantes de uma escola da rede de ensino público. O grupo de adultos jovens e de idosos foi composto por indivíduos da população em geral.

\section{Material}

Os estímulos foram apresentados em um monitor Samsung (17 polegadas), com varredura da tela ajustada para 60 $\mathrm{Hz}$, acoplado a um microcomputador modelo IBM-PC. O controle da apresentação dos estímulos e o registro do tempo de resposta foram realizados pelo utilitário E-Prime 2.0 (Schneider, Eschman, \& Zuccoloto, 2007). A luminância dos estímulos foi mensurada com um fotômetro modelo HCT99 D acoplado a um detector LDM-99 (Gigahertz-Optik).

\section{Estímulos e Procedimento}

Os estímulos foram apresentados em preto com uma luminosidade de $0,03 \mathrm{~cd} / \mathrm{m}^{2}$ sobre um fundo branco com uma luminosidade de $346,625 \mathrm{~cd} / \mathrm{m}^{2}$. Os participantes mantiveram os olhos a uma distância aproximada de $57 \mathrm{~cm}$ da tela de apresentação sem restrições aos movimentos da cabeça.

Cada prova era iniciada com a apresentação de um ponto de fixação que permanecia no centro da tela por 1000 ms. Imediatamente após este intervalo, um arranjo central composto por 2,4 ou 6 letras em preto $\left(1^{\circ} \mathrm{x} 1^{\circ}\right.$ graus de ângulo visual) e dispostas em um círculo imaginário de raio igual a $2,5^{\circ}$ graus de ângulo visual era apresentado. Este 
arranjo sempre era acompanhado pela apresentação de uma letra flanqueadora $\left(2^{\circ} \times 2^{\circ}\right.$ graus de ângulo visual $)$, também apresentada em preto e disposta a $7^{\circ}$ graus de ângulo visual à direita ou a esquerda do ponto central da tela.

Uma letra alvo, que poderia ser um X ou um N, sempre era apresentada aleatoriamente em uma das seis posições possíveis no arranjo. A letra alvo era apresentada acompanhada de uma, três ou cinco letras distratoras (Z, K, Y, V ou H), em diferentes posições no círculo imaginário (carga perceptual 2 , 4 e 6 respectivamente). Simultaneamente à apresentação das letras do arranjo central, uma letra flanqueadora incompatível ( $\mathrm{N}$, quando a letra alvo no arranjo central era $\mathrm{X}$ ou $\mathrm{X}$ quando a letra no arranjo central era $\mathrm{N}$ ) ou neutra (L ou T) em relação ao universo das letras alvo era apresentada.

A tarefa do participante era identificar a letra alvo o mais rápido possível pressionando uma tecla do teclado numérico correspondente a cada letra alvo (número 1 do teclado numérico do computador para a letra X e 2 para a N). O participante era informado que esta letra flanqueadora era irrelevante para a sua tarefa e deveria ser ignorada ao longo das provas. Foi solicitado aos participantes que mantivessem o olhar fixo no centro da tela. A apresentação dos estímulos imperativos era restrita a $500 \mathrm{~ms}$ para os grupos compostos por crianças e adultos. O tempo de apresentação dos estímulos imperativos não foi restrito para o grupo composto por idosos. Após cada resposta um sinal de mais "+" era apresentado por 1 segundo como feedback da resposta do participante $(\mathrm{azul}=$ correto, vermelho $=$ incorreto $)$. Após este intervalo uma nova prova era apresentada. A Figura 1 mostra um exemplo esquemático dos estímulos a serem utilizados e da seqüência de eventos em cada prova.

\section{Resultados}

\section{Tempo de Reação}

A análise da variância (ANOVA) foi realizada com a média do tempo de reação levando em consideração os seguintes fatores: Grupo Etário (crianças x adultos x idosos), compatibilidade do flanqueadora (neutro e incompatível), e carga perceptual $(2,4,6)$. De acordo com esta análise houve um efeito significativo no tempo de reação em função do grupo etário $\mathrm{F}(2,18)=18,904 ; \mathrm{p}<0,01, \eta^{2}=0,67$, grupo de crianças $(\mathrm{M}=1074 \mathrm{~ms}, \mathrm{DP}=133)$; grupo de adultos $(\mathrm{M}=$ $817 \mathrm{~ms}$, DP = 105); grupo de idosos $(\mathrm{M}=1245 \mathrm{~ms}$, DP = 242). Os adultos jovens compuseram o grupo mais rápido em comparação aos grupos formados por idosos e crianças. A carga perceptual apresentou um efeito significativo $\mathrm{F}(2,18)=$ $111,933, p<0,01, \eta^{2}=0,93$, expresso em um aumento linear do TR em função do aumento da carga perceptual $(2,4,6)$, com os seguintes TR médios: Carga $2(\mathrm{M}=879 \mathrm{~ms}$, DP = $158)$; carga $4(\mathrm{M}=1029 \mathrm{~ms}, \mathrm{DP}=193)$; $\operatorname{carga} 6(\mathrm{M}=1228$ $\mathrm{ms}, \mathrm{DP}=299$ ). O fator compatibilidade da letra flanqueadora apresentou uma diferença significativa entre as condições investigadas (neutra e incompatível), $\mathrm{F}(1,9)=4,945 ; \mathrm{p}=$ $0,05, \eta^{2}=0,35$, expressa em um aumento significativo do TR na condição letra flanqueadora incompatível, TR médio na condição letra flanqueadora neutra $=1032 \mathrm{~ms}$; TR médio na condição letra flanqueadora incompatível $=1059 \mathrm{~ms}$.

A interação entre os fatores idade e compatibilidade da letra flanqueadora foi significativa $F(2,18)=7,550 ; p=$ $0,01, \eta^{2}=0,46$. Os fatores idade e carga perceptual também apresentaram interação significativa, $\mathrm{F}(4,36)=10,62 ; \mathrm{p}<$ $0,01 \eta^{2}=0,54$. A interação entre compatibilidade da letra flanqueadora e carga perceptual foi significativa $\mathrm{F}(2,8)=$ 8,$512 ; \mathrm{p}<0,01, \eta^{2}=0,49$. Não houve interação significativa entre os três fatores grupo etário $\mathrm{x}$ compatibilidade $\mathrm{x}$ carga $\mathrm{F}(4,36)=0,389 ; \mathrm{p}=0,815 \eta^{2}=0,04$.

O efeito no tempo de reação em função da carga perceptual corrobora o modelo proposto por Lavie (1995) no qual a interferência da letra flanqueadora incompatível é maior na condição de baixa carga perceptual em comparação a condição de alta carga perceptual. Este efeito é calculado da seguinte maneira: TR Incompatível ${ }_{(\text {carga } 2,4,6)}$ - TR Neutro (carga 2,4,6). A Figura 2 mostra este efeito em função do grupo etário.

Como mostra a Figura 2 o efeito da carga perceptual apresenta efeitos distintos em função do grupo etário $\mathrm{F}(2,18)=$ 7,$55 ; \mathrm{p}<0,01$ e em função da carga perceptual $\mathrm{F}(2,18)=8,51$; $\mathrm{p}<0,01$. Não houve interação entre os fatores grupo etário e carga perceptual $\mathrm{F}(4,36)=0,38 ; \mathrm{p}=0,81$. Esta independência entre os fatores analisados pode sugerir um processamento
Fixação

$1000 \mathrm{~ms}$

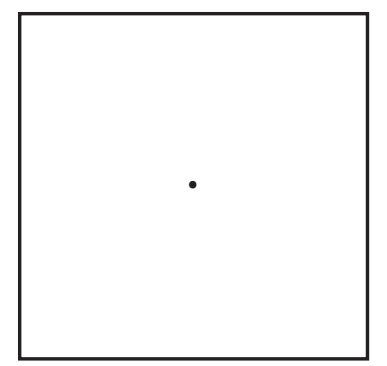

$200 \mathrm{~ms}$

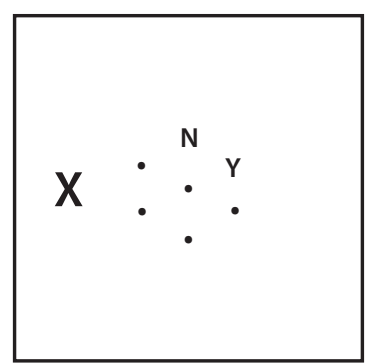

1500

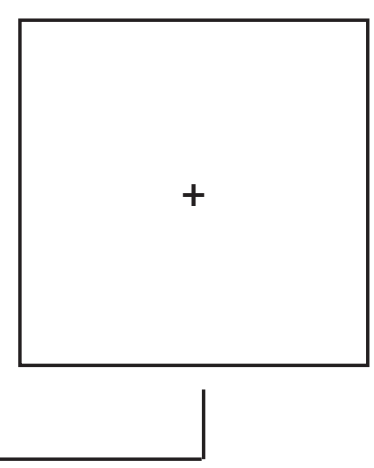

Figura 1. Representação esquemática do display do experimento 2. 
similar nos três grupos investigados. Uma análise em separado (ANOVA) mostrou que o efeito da letra flanqueadora foi maior no grupo formado por idosos na condição de baixa carga perceptual (2) $\mathrm{F}(2,18)=4,78 ; \mathrm{p}<0,05$.

Uma questão a ser investigada é se o efeito no tempo de reação pode ser atribuído apenas a uma lentidão geral no processamento, característico em idades mais avançadas (Salthouse, 1996) ou se os padrões observados de processamento são específicos às grupos etários investigados (West, 1996). Para tanto, foi utilizado o procedimento proposto por Maylor e Lavie (1998) no qual o efeito de compatibilidade é calculado de maneira proporcional aos tempos de reação de linha de base (letras flanqueadoras neutras). Assim, o efeito no TR é calculado através do seguinte procedimento: (TR incompatível - TR neutro) / TR neutro, como apresentado na Figura 3. Esta análise mostrou o mesmo padrão dos resultados observados com o tempo de reação.

\section{Análise do erro}

A média da porcentagem de erros cometidos em cada condição foi analisada por meio de uma ANOVA. Esta análise confirmou um efeito significativo do fator grupo etário na média de erros cometidos, crianças $(12,8 \%)$, adultos $(2,8 \%)$ e idosos $(5,62 \%) \mathrm{F}(2,18)=13,28 ; \mathrm{p}<0,01$.

A média de erros cometidos também foi significativa em função da compatibilidade da letra flanqueadora, Neutra $(6,25 \%)$ e Incompatível $(7,93 \%) \mathrm{F}(1,9)=6,21 ; \mathrm{p}<0,05$. A porcentagem de erro também foi significativamente distinta em função da carga perceptual, dois $(5,88 \%)$, quatro $(5,77 \%)$ e seis $(9,63 \%)$. Houve interação significativa entre os fatores grupo etário e carga perceptual $\mathrm{F}(4,36)=7,6 ; \mathrm{p}<0,01$, como mostra a Figura 4.

As interações entre grupo etário e compatibilidade, compatibilidade e carga perceptual e a tripla interação não

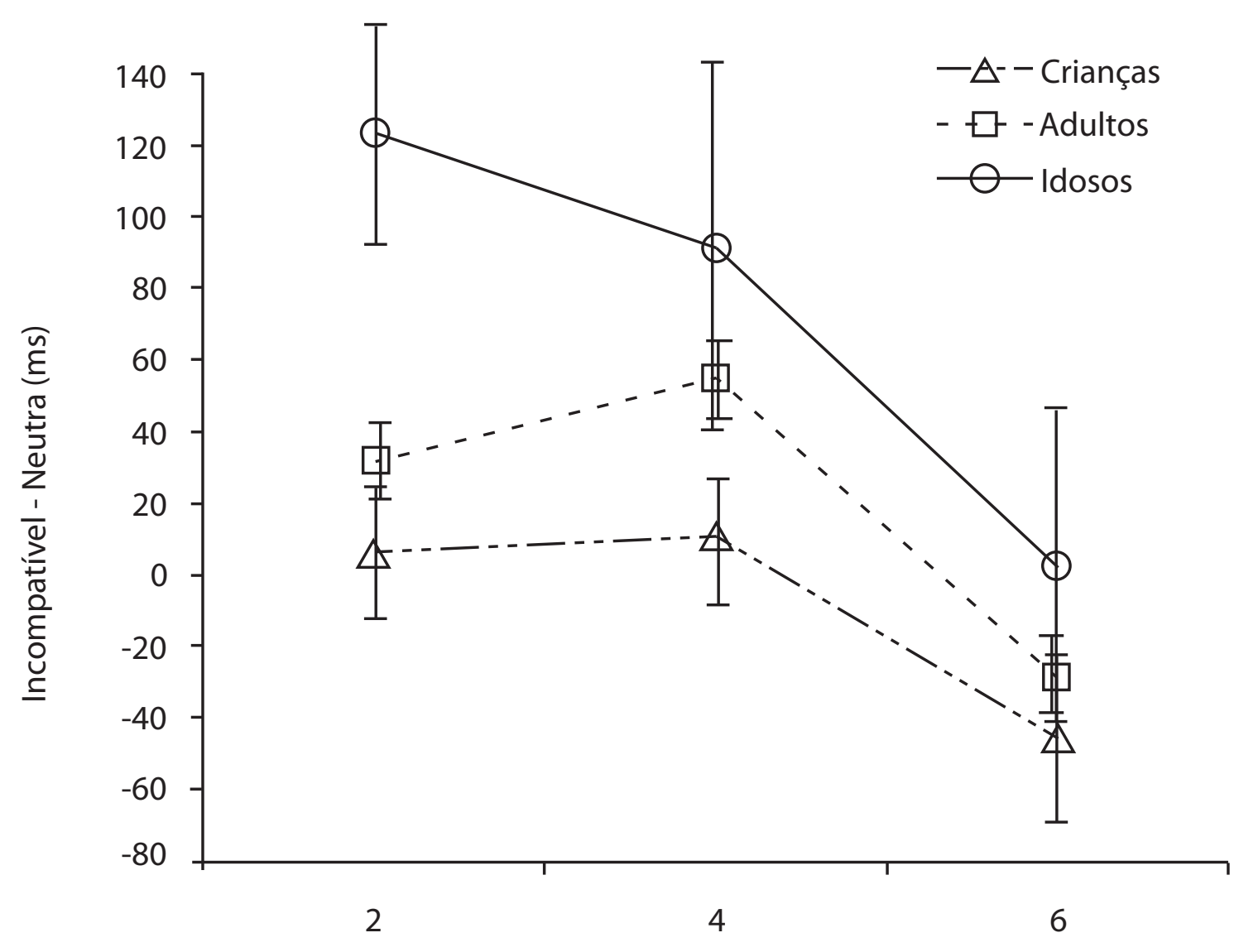

Carga Perceptual

Figura 2. Diferença do TR médio em milissegundos entre as condições incompatível e neutra em função da carga perceptual e do grupo etário. As barras de dispersão mostram o erro padrão de cada grupo. 


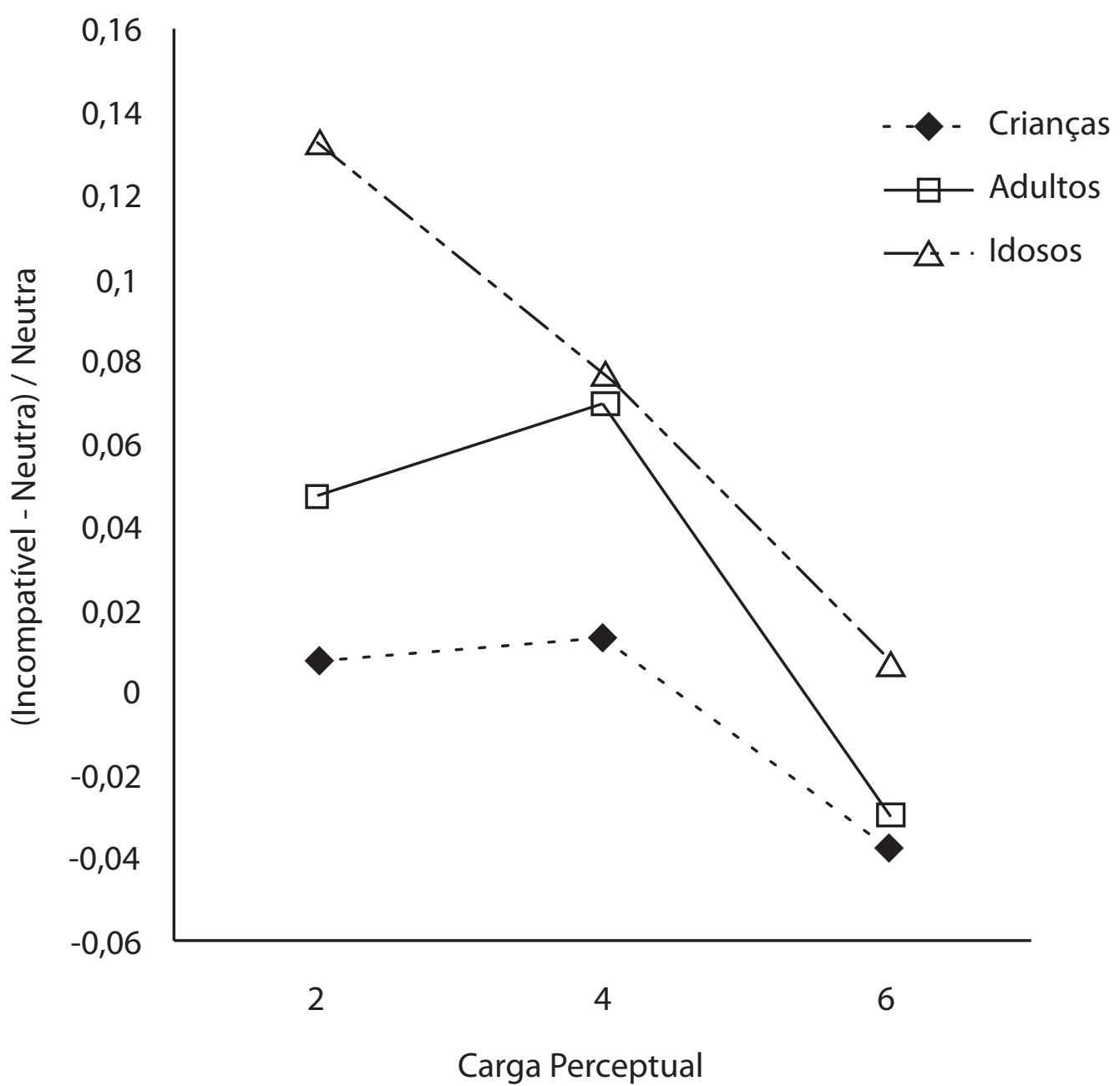

Figura 3. TR do efeito da compatibilidade [(TR incompatível /TR neutro)/ TR neutro] em função da carga perceptual.

foram significativas ( $\mathrm{p}<1$ ). A Figura 5 mostra a distribuição geral da porcentagem de erros cometidos. Uma análise em separado que avaliou o impacto do erro em função da carga perceptual (baixa ou alta) e dos grupos etários, evidenciou uma quantidade de erros maior na presença da letra flanqueadora incompatível no grupo de idosos $(9,14 \%)$ e crianças $(8,2 \%)$ em condições de baixa carga perceptual (2). Nesta condição de carga o grupo de crianças apresentou também uma grande quantidade de erros nas condições com letra flanqueadora neutra $(7,74 \%)$. Ainda nas condições com baixa carga perceptual o grupo de adultos [incompatível $(3,65 \%)$ e neutro $(2,85 \%)$ ] e idosos [neutro $(3,69 \%)$ ] apresentaram melhor acurácia.

Nas condições com alta carga perceptual o grupo composto por crianças apresentou uma porcentagem elevada de erros, tanto nas condições com letra flanqueadora neutra $(17,6 \%)$ quanto incompatível (19,9\%) em comparação à porcentagem de erros cometidos pela população de adultos [neutra $(4,2 \%)$, incompatível $(3,81 \%)$ ] e idosos [neutra $(4,38 \%)$, incompatível $(8,08 \%)$ ], como mostra a Figura 6 .

\section{Discussão}

De acordo com a teoria da carga perceptual a quantidade, ou carga, de informação a ser processada pelo sistema visual é determinante para o tipo de seleção a ser implementada pelo sistema cognitivo. Esta teoria preconiza que em condições de alta carga perceptual a seleção da informação é realizada com base nas características físicas dos estímulos o que caracteriza um processo de seleção precoce da informação. Por outro lado, em condições de baixa carga perceptual a informação seria selecionada através de um processo de análise semântica caracterizado por uma seleção tardia ( Lavie, 1995; Lavie \& Cox, 1997; Lavie \& Tsal, 1994). O objetivo principal deste trabalho foi investigar o mecanismo de seleção atentivo baseado na carga perceptual em três grupos etários compostos por crianças, adultos jovens e idosos.

Como preconizado pela teoria da carga perceptual, os resultados evidenciaram um efeito deletério no tempo de reação, causado pela letra flanqueada, maior em condições de baixa carga perceptual em comparação às condições de 


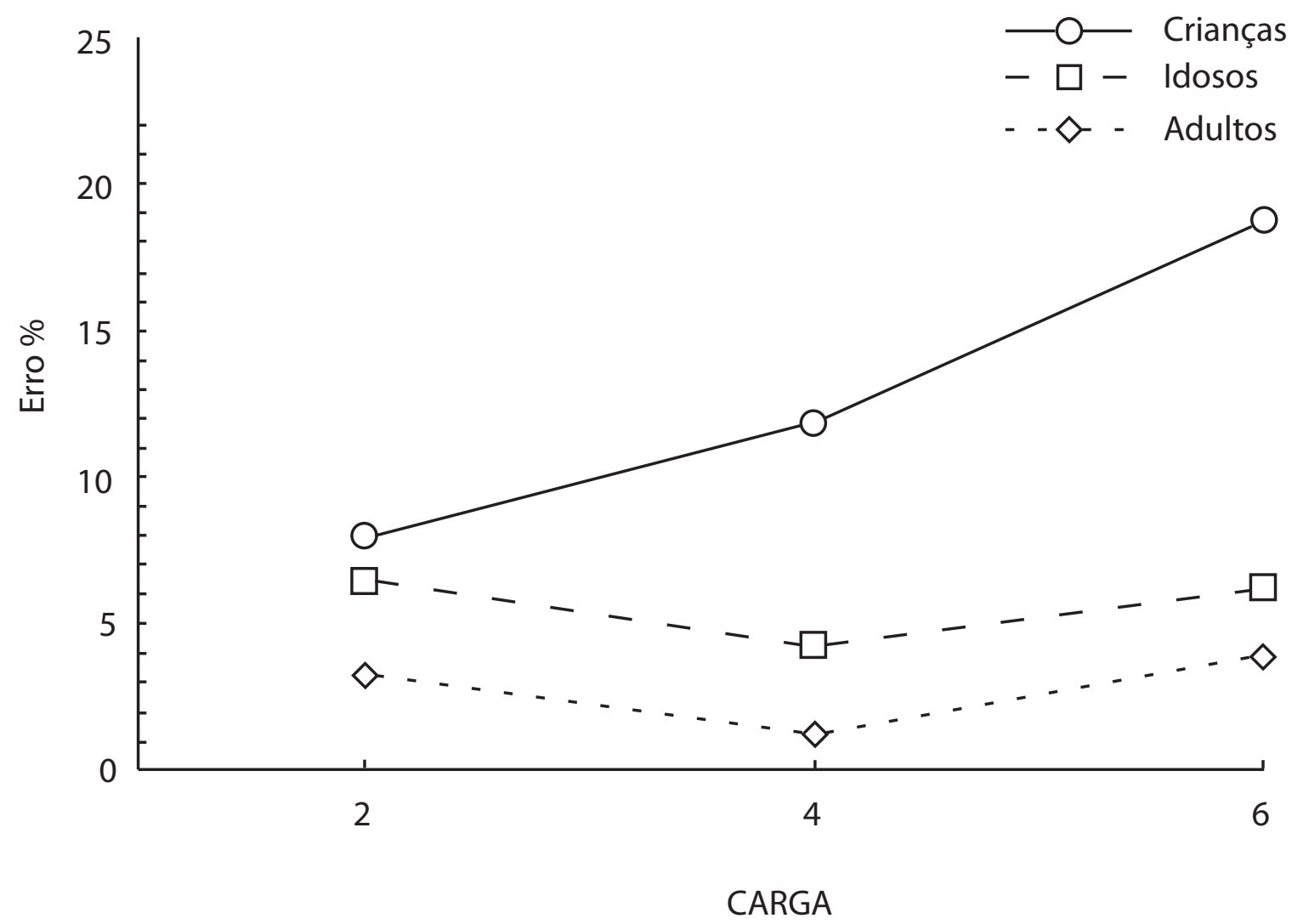

Figura 4. Porcentagem de erro cometidos em cada grupo etário em função da carga perceptual.

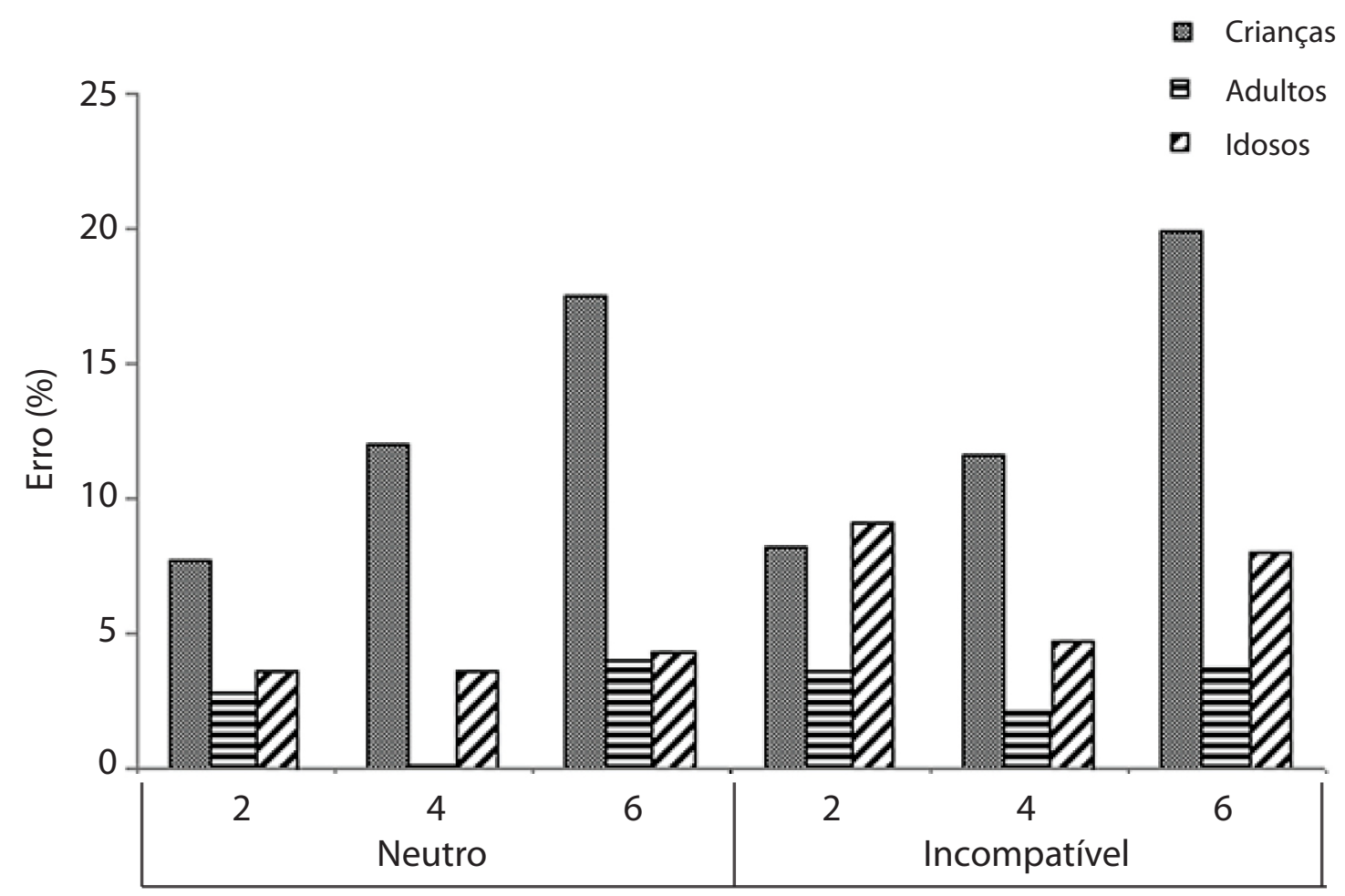

Figura 5. Distribuição geral da porcentagem de erros cometidos em todas as condições experimentais (grupo etário $\mathrm{x}$ carga perceptual x compatibilidade da letra flanqueadora). 


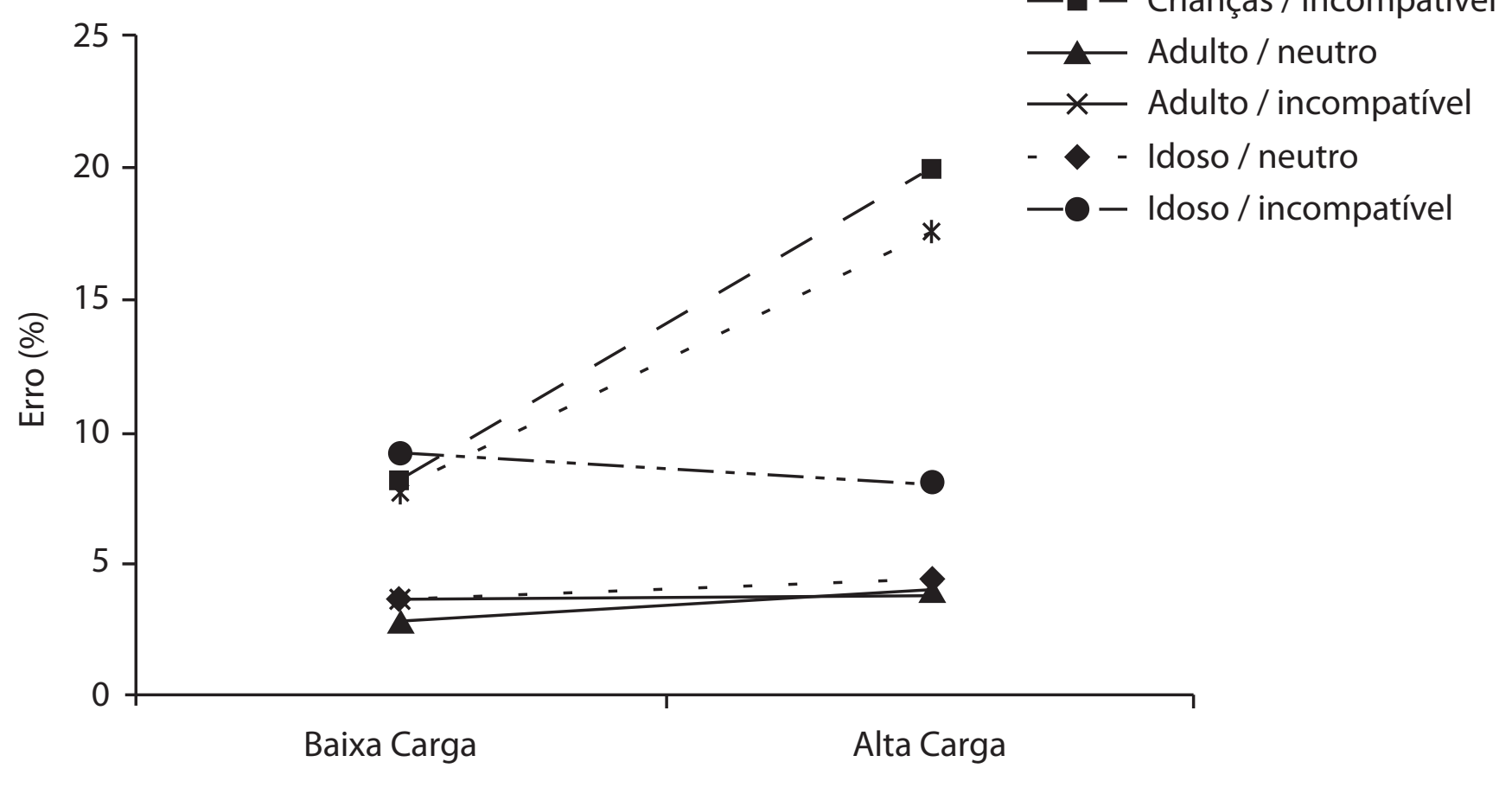

- * - Crianças / neutro

- - Crianças / incompatível

$\longrightarrow$ Adulto / neutro

$\longrightarrow$ Adulto / incompatível

$\checkmark$ - Idoso / neutro

- Idoso / incompatível

Figura 6. Porcentagens de erro nos três grupos etários em função da carga perceptual (alta e baixa carga) nas condições letra flanqueadora incompatível e neutra.

alta carga perceptual nos três grupos etários investigados. Assim como em resultados observados por outros autores (Lavie \& Cox, 1997; Lavie \& Fox 2000; Maylor \& Lavie, 1998) o efeito da letra flanqueadora é mantido até a carga perceptual composta por quatro elementos relevantes, diminuindo rapidamente nas condições com carga perceptual composta por seis elementos. Mais especificamente, acerca de possíveis diferenças no desenvolvimento dos mecanismos atentivos nos grupos investigados, este experimento sugere uma independência entre o fator grupo etário e carga perceptual o que pode indicar um processamento comum aos três grupos investigados apesar de uma maior lentidão na seleção da informação no grupo composto por idosos.

A análise do erro cometido pelos participantes mostrou que em condições de baixa carga perceptual o grupo composto por crianças (letra flanqueadora neutra e incompatível) e idosos (letra flanqueadora incompatível) apresentou uma porcentagem de erros semelhantes, enquanto que nas condições de alta carga perceptual apenas o grupo composto por crianças apresentou uma porcentagem elevada de erros, o que pode sugerir uma imaturidade do sistema seletivo na população de crianças. Isto pode indicar que o grupo composto por crianças ainda não atingiu a maturidade do sistema de seleção precoce.

Por outro lado, o aumento da taxa de erros no grupo composto por idosos em comparação ao grupo composto por adultos jovens, na condição de baixa carga perceptual, pode indicar uma perda de eficiência no sistema de seleção tardia da informação nesta população. Estes resultados vão ao encontro da interpretação de momentos distintos de maturação dos sistemas de seleção precoce e tardia e sugerem uma dinâmica de manutenção destes recursos peculiar a cada fase do desenvolvimento como sugerido inicialmente por Plude, Enns e Brodeur (1994) e Huang-Pollock, Carr e Nigg (2002).

Em conjunto, os nossos resultados corroboram a teoria da carga perceptual e sugerem padrões específicos de eficiência na seleção da informação relevante e inibição da informação irrelevantes nos três grupos etários investigados.

\section{Referências:}

Broadbent, D. E. (1958). Perception and communication. New York: Pergamon Press.

Cerella, J. (1985). Information processing rates in the elderly. Psychological Bulletin, 98, 1, 67-83.

Deutsch, J. A., \& Deutsch, D. (1963). Attention: Some theoretical considerations. Psychological Review, 70, 80-90.

Hasher, L., \& Zacks, R. T. (1988). Working memory, comprehension, and aging: A review and a new view. In G, H. Bower (Ed.), The psychology of learning and motivation, 22, 193-225.

Huang-Pollock, C. L., Carr, T. H., \& Nigg, J. T. (2002). Development of selective attention: Perceptual load influences early versus late attentional selection in children and adults. Developmental Psychology, 38, 363-375.

Lavie, N. (1995). Perceptual load as a necessary condition for selective attention. Journal of Experimental Psychology: Human Perception and Performance, 21, 451-468.

Lavie, N., \& Cox, S. (1997). On the efficiency of visual selective attention: Efficient visual search leads to inefficient distractor rejection. Psychological Science, 8, 395-398. 
Lavie, N., \& Tsal, Y. (1994). Perceptual load as a major determinant of the locus of selection in visual attention. Perception \& Psychophysics, 56, 183-197.

Maylor, E. A., \& Lavie, N. (1998). The influence of perceptual load on age differences in selective attention. Psychology and Aging, 13, 563-573

Pesce, C., Guidetti, L., Baldari, C., Tessitore, A., \& Capranica, L. (2005). Effects of aging on visual attentional focusing. Gerontology, 51, 266-276.

Plude, D. J, Enns, J. T., \& Broudeur, D. (1994) The development of selective attention: A life-span overview. Acta Psychologica, $86,227-272$.

Salthouse, T. A. (1996). The processing-speed theory of adult age differences in psychological cognition. Review Copyright, 103, 403-428.
Schneider, W., Eschman, A. \& Zuccoloto, A. (2007). (Software) E-prime 2.0. Psychological Software Inc.

Treisman, A. M. (1964). Selective attention in man. British Medical Bulletin, 20, 12-16.

West, R. (1996). An application of prefrontal cortex function theory to cognitive aging. Psychological Bulletin, 120, 272-292.

Recebido em 10.07.2009

Primeira decisão editorial em 22.12.2009

Versão final em 22.01.2010

Aceito em 03.02.2010

\section{CONGRESO PSICOLOGÍA Y RESPONSABILIDAD SOCIAL: ABORDAJES INTERDISCIPLINARIOS}

Período: 07 de Abril - 09 Abril 2011

Local: Bogotá, CO

\section{CONGRESSO BRASILEIRO DE TERAPIAS COGNITIVAS "DA PSICOPATOLOGIA À SAÚDE MENTAL POSITIVA"}

Período: 06 de Abril - 09 Abril 2011

Local: Florianópolis - SC, BR 